\title{
Longitudinal stability in multiharmonic standing wave linacs
}

\author{
L. R. Carver" ${ }^{*}$ and R. M. Jones \\ School of Physics and Astronomy, The University of Manchester, \\ Oxford Road, Manchester M13 9PL, United Kingdom, \\ and Cockcroft Institute of Science and Technology, Daresbury WA4 4AD, United Kingdom \\ Y. Jiang and J. L. Hirshfield \\ Omega-P, Inc., 258 Bradley Street, New Haven, Connecticut 06510, USA, \\ and Physics Department, Yale University, New Haven, Connecticut 06520-8120, USA
}

(Received 23 April 2016; published 22 September 2016)

\begin{abstract}
Accelerating cavities that excite multiple modes at integer harmonics of the fundamental frequency have the potential to be used to suppress the onset of rf breakdown and reduce the pulsed surface heating at high accelerating gradients. Understanding the effect of an additional harmonic cavity mode on the longitudinal beam dynamics is important to their development and use. A Hamiltonian that describes the longitudinal motion of a particle as it traverses a chain of multiharmonic cavities has been derived and is applied to the case of a second harmonic cavity. The Hamiltonian is based upon formalisms found in literature for the fundamental harmonic and is extended to include different longitudinal field distributions and harmonic frequencies. The study initially explores the longitudinal motion for moderate accelerating gradients with high- $\beta$ protons, as this will allow fundamental properties of the stable region (acceptance and shape of the rf bucket) to be determined. High accelerating gradients are also investigated but the focus will be on phase stability throughout. This work concludes by considering the longitudinal dynamics of a modified European Spallation Source accelerator, comprised of multiharmonic cavities that has specifications broadly consistent with the accelerator.
\end{abstract}

DOI: 10.1103/PhysRevAccelBeams.19.094001

\section{INTRODUCTION}

High gradient electric fields are used to accelerate charged particles. The accompanying intense electric and magnetic fields on the surface of the cavity [1,2] can cause rf breakdown and give rise to pulsed surface heating [3]. Radio-frequency breakdown is a complex phenomenon, and the role of the magnetic field in a breakdown is an active area of study, however it is believed that surface field emission and surface heating could both be precursors to a breakdown event. Accelerating cavities that can support multiple harmonically related eigenmodes could be used to deepen the understanding of rf breakdown and pulsed surface heating at high accelerating gradients [4], both for CLIC-like applications [5-7], as well as applications for other linear colliders [8].

Multiharmonic cavities have unconventional surface electric and magnetic field profiles that can potentially lower the surface field emission and/or pulsed surface heating without compromising the gradient. Two particular phenomena found in multiharmonic cavities provide the

\footnotetext{
*Lee.Robert.Carver@cern.ch

Published by the American Physical Society under the terms of the Creative Commons Attribution 3.0 License. Further distribution of this work must maintain attribution to the author(s) and the published article's title, journal citation, and DOI.
}

main motivation for their use: (a) the anode-cathode effect [4], which can be found in an asymmetric multiharmonic cavity that is designed such that the cathode fields are significantly smaller than the anode fields. This effect will raise the work function barrier to suppress field and secondary electron emission, and (b) a reduction in the surface heating by lowering the average $H_{\|}^{2}$ along the surface [9]. Experiments are currently under way that aim to provide an experimental basis to these effects [10-12].

Accelerators have long been utilizing harmonic rf systems to improve the quality and lifetime of particle bunches $[13,14]$. However, these rf systems rely on the fabrication of additional cavities operating with a fundamental frequency that is a factor of $h$ larger than the main accelerating cavities [15], where $h$ is the harmonic number. The phase of the harmonic cavity can be adjusted such that the bunch sees a linear field accelerating field [16]. This reduces the energy spread and lengthens the bunch, allowing for much higher beam lifetimes. This is particularly important for the European XFEL project [17], for example, where the energy spread reduction can result in a laser with much higher brilliance.

For a cavity that excites multiple harmonic modes to be used in an accelerator, the combined effect of the fundamental and harmonic mode on the longitudinal dynamics needs to be explored. To achieve this, a Hamiltonian is derived that describes the behavior of particles with 
deviations from a particle with idealized phase and energy (the so-called synchronous particle). Multiharmonic accelerating cavities have additional harmonic modes present that follow different (and higher order) longitudinal profiles. The formalisms currently found in literature $[18,19]$ will need to be generalized and extended in order to model these types of cavity.

In this study we derive the general Hamiltonian for a standing wave (SW) multiharmonic cavity that excites a $\mathrm{TM}_{010}$ mode with any higher harmonic mode. The Hamiltonian will be applicable to a variety of modal configurations, and will describe the longitudinal motion of particles as they traverse a linear accelerator comprising a chain of multiharmonic cavities. Protons with initial energies of several $\mathrm{GeV}$ will be used throughout. Two cases will be studied: first for small accelerating gradients of approximately $10 \mathrm{kV} / \mathrm{m}$. This allows the general features of the stable accelerating region to be determined. Then high accelerating gradients of approximately $10 \mathrm{MV} / \mathrm{m}$ will be applied, in order to better understand how this work could be applied to specific linacs.

The next section outlines the model for a single mode cavity using the well-known Hamiltonian. Section III will derive a general-purpose Hamiltonian for a particle traversing a cavity that can excite two harmonic modes. In Sec. IV, this Hamiltonian is applied to a cavity that consists of a fundamental accelerating $\mathrm{TM}_{010}$ mode with a second harmonic $\mathrm{TM}_{011}$ mode. The relative phase between the two modes will be varied, while maintaining a constant accelerating gradient for the synchronous particle. Both lowgradient and high-gradient acceleration will be explored. This is followed in Sec. V by an application of the Hamiltonian to an ESS-like cavity linac, in order to show that cavities of this type can accelerate bunches with parameters that are readily available. Comparisons to results from tracking simulations will also be shown. The final section provides some concluding remarks on this study.

The work that is presented in this paper is applicable for rf driven multiharmonic cavities. A linear accelerator setup of this kind allows the amplitude and phase of the harmonic field to be tuned, providing greater control over the longitudinal dynamics. However, this work could also be applied to a beam-driven collinear two beam accelerator. In this setup, it is preferable to use a third harmonic cavity (or any odd longitudinal distributions) over a second harmonic (or any even longitudinal distribution) in order to enhance the overall energy gain [4].

The anticipated benefit from surface heating reduction and prevention of $\mathrm{rf}$ breakdown reduces with increasing harmonic number [12]. This is because $\Delta T \propto$ $\left\langle H_{1}^{2}\right\rangle\left[(1-\alpha)^{2}+\alpha^{2} \eta^{2}\right]$, where $\Delta T$ is the temperature rise on the surface of the cavity, $\alpha$ is the percentage contribution to the total accelerating field from the harmonic mode, $\left\langle H_{1}^{2}\right\rangle$ is the average over one cycle of the magnetic field from the fundamental mode on the surface squared, and $\eta \approx \frac{H_{h}^{\max }}{H_{1}^{\max }}$ is the ratio of the strength of the harmonic magnetic field to the fundamental. As $\eta$ approaches 1, the strength of the magnetic field of the harmonic mode is approaching the strength of the magnetic field of the fundamental mode. For higher harmonics the value of $\eta$ increases, and the maximum possible temperature reduction reduces. Therefore, we only consider the case where $h=2$ here.

\section{SINGLE MODE HAMILTONIAN}

The multiharmonic Hamiltonian is an extension of the single mode analysis. Therefore it is important to describe the single mode case in order to understand how the additional harmonic perturbs the rf bucket. The following section provides a summary of work on the single mode Hamiltonian originally found in Refs. $[18,19]$.

For an infinite chain of SW cavities, the longitudinal profile is $\cos (k z)$ for a $\mathrm{TM}_{010}$ mode where $z$ is the longitudinal position within the cavity which is centered on $z=0, k=\frac{2 \pi}{\beta_{s} \lambda}, \beta_{s}$ is the normalized velocity of the synchronous particle and $\lambda$ is the wavelength of the fundamental mode. The synchronous particle is a particle that has the ideal phase and energy at any given position. The derived Hamiltonian governs the longitudinal motion of any particle traversing this cavity chain relative to the synchronous particle. In order to achieve this, equations concerning the evolution of the phase and energy of a particle with respect to the longitudinal coordinate $s$ must be determined.

For a $\pi$-mode SW cavity, the phase advance of the rf field from one cavity to the next is given by

$$
\phi_{n}=\phi_{n-1}+\omega \frac{g}{\beta_{n-1} c},
$$

where

$$
g=\frac{\beta_{s, n-1} \lambda}{2}
$$

is the cavity gap, $c$ is the velocity of light and $\omega$ is the angular frequency of the mode. The subscripts $s$ and $n$ refer to the synchronous particle and the cavity number, respectively. From this, the phase advance of a particle relative to the synchronous particle from cavity $(n-1)$ to cavity $n$ is given by

$\Delta\left(\phi-\phi_{s}\right)_{n}=\Delta \phi_{n}-\Delta \phi_{s, n}=\pi \beta_{s, n-1}\left[\frac{1}{\beta_{n-1}}-\frac{1}{\beta_{s, n-1}}\right]$.

Applying a Taylor expansion for a small perturbation about $\beta_{s}$ gives

$$
\frac{1}{\beta}-\frac{1}{\beta_{s}}=\frac{1}{\beta_{s}+\delta \beta}-\frac{1}{\beta_{s}} \approx \frac{\delta \beta}{\beta_{s}^{2}},
$$


where the change in velocity $d \beta$ across one cavity is given by $\delta \beta=\delta W / m c^{2} \gamma_{s}^{3} \beta_{s}, m$ is the mass of the particle and $\gamma_{s}$ is the relativistic gamma factor. This leads to the difference equation that shows how the particles phase evolves with cavity number. The energy gain of a particle also needs to be in terms relative to the synchronous particle. For simplicity this term is normalized to the rest energy of particle under study. It therefore follows that the substitution $w=\delta W / m c^{2}=\left(\Delta W_{n}-\Delta W_{s, n}\right) / m c^{2}$ can be made. The phase difference equation is now given by

$$
\Delta\left(\phi-\phi_{s}\right)_{n}=-\pi \frac{w}{\gamma_{s, n-1}^{3} \beta_{s, n-1}^{2}} .
$$

The SW electric field of a $\mathrm{TM}_{010}$ mode is a combination of the field profile in the $z$-direction and the oscillation at a specific frequency, given by

$$
E_{T}=E_{0} \cos (k z) \cos (\omega t+\phi),
$$

where $\phi$ is the phase of the field when the particle is at $z=0$ and $E_{0}$ is the field amplitude. For a particle moving with $t=z / \beta c$, the energy gain of a particle as it crosses a single cavity is given by

$$
\begin{aligned}
W & =q E_{0} \int_{-g / 2}^{g / 2} \cos (k z) \cos (\omega t+\phi) d z \\
& =q V_{0} T(\beta) \cos (\phi),
\end{aligned}
$$

where $V_{0}$ is the axial rf voltage, given by

$$
V_{0}=E_{0} \int_{-g / 2}^{g / 2} \cos (k z) d z
$$

and $T(\beta)$ is the transit time factor, which is determined by

$$
\begin{aligned}
T(\beta)= & \frac{E_{0} \int_{-g / 2}^{g / 2} \cos (k z) \cos \left(\frac{\omega z}{\beta c}\right) d z}{V_{0}} \\
& -\tan (\phi) \frac{E_{0} \int_{-g / 2}^{g / 2} \cos (k z) \sin \left(\frac{\omega z}{\beta c}\right) d z}{V_{0}} .
\end{aligned}
$$

The difference equation for the energy of the particle with respect to the synchronous particle is

$$
\Delta W_{n}-\Delta W_{s, n}=q g E_{1} T(\beta)\left[\cos \left(\phi_{n}\right)-\cos \left(\phi_{s}\right)\right],
$$

where $E_{1}=V_{0} / g$ is the accelerating gradient.

Equations (5) and (11) can be written as a continuous function, rather than having the discrete action of the particles at the center of each cavity. Using $n=2 s / \beta_{s} \lambda$ they can be expressed as

$$
\frac{d \phi}{d s}=-2 \pi \frac{w}{\gamma_{s}^{3} \beta_{s}^{3} \lambda}=\frac{\partial H}{\partial w}
$$

and

$$
\frac{d w}{d s}=q \frac{E_{1} T(\beta)}{m c^{2}}\left[\cos (\phi)-\cos \left(\phi_{s}\right)\right]=-\frac{\partial H}{\partial \phi} .
$$

As $\phi$ and $w$ are variables canonically dependant on $s$, a Hamiltonian can be constructed that describes the particle motion in phase space, which is given as

$H=-\frac{\pi}{\beta_{s}^{3} \gamma_{s}^{3} \lambda} w^{2}-q \frac{E_{1} T(\beta)}{m c^{2}}\left[\sin (\phi)-\phi \cos \left(\phi_{s}\right)\right]$.

This is consistent with the usual form of a Hamiltonian $H=K+V$ where $K$ is the kinetic energy and is represented by the $w$-dependent term (first term in the expression) and $V$ is the potential represented by the $\phi$-dependent term (second term in the expression). A particle that has a deviation in phase or energy will orbit the synchronous particle in phase space according to the Eqs. (12) and (13). A particle orbit is found by plotting the $\phi$ and $w$ points for a set of initial coordinates. For increasingly large deviations, the particles orbit becomes wider and wider, until eventually it becomes unstable. A stable orbit is one that follows a closed curve, whereas an unstable orbit is an open curve that trails off giving rise to high energy and phase differences. It is important to know where the boundary between stable and unstable orbits lie. This boundary is known as the separatrix and it can be determined by two points, the stable fixed point and the minimum phase for stable motion. The stable fixed point lies at $\left(\phi=-\phi_{s}\right.$, $w=0$ ). This is the maximum phase difference a particle can have because at this point the particle will be gaining the same energy as the synchronous particle, therefore no phase space motion is expected. The minimum phase for stable motion lies at zero kinetic energy $\left(\phi=\phi_{2}, w=0\right)$, which arises when the potential at this point is the same as for the stable fixed point.

To calculate the value of the Hamiltonian on the separatrix, $\phi_{2}$ needs to be determined. This is found by equating the value of the potential at the minimum and maximum phase boundaries. The potential at these points are the same, i.e.

$$
V\left(\phi=\phi_{2}\right)=V\left(\phi=-\phi_{s}\right)
$$

Figure 1 shows typical profiles pertaining to a $2 \mathrm{GeV}$ proton that experiences an accelerating gradient of $E_{0}=10 \mathrm{kV} / \mathrm{m}$. The uppermost plot is the energy gain of a particle across one cavity as a function of the initial phase offset $\phi$. The middle plot is the potential of the cavity on the separatrix, where it can be seen that a potential well exists in the region of stability. The lower plot shows the 




FIG. 1. Top: Energy gain as a function of phase offset for a particle traversing a single cavity. Middle: Potential for separatrix. Bottom: Phase space contours in black, separatrix in red and stable orbits in blue.

particle trajectory in phase space, which has been solved for various initial phase offsets and covers both stable and unstable orbits, including the separatrix. The acceptance of a cavity is determined by calculating the area of the rf bucket, and refers to the maximum bunch emittance that can undergo stable acceleration. The conventional unit of longitudinal acceptance is $[\mathrm{eV}][\mathrm{s}]$, however here normalized energy is being used. Therefore, the acceptance of the separatrices shown here are in units of radians (which is related to time through the fundamental frequency). Henceforth, for convenience when referring to the normalized acceptance, the term acceptance will be used.

In order to ensure the derived Hamiltonian is accurate, a particle tracker based on Eq. (7) was created that numerically tracks a single particle through a chain of cavities for a given offset $\phi$ and initial energy deviation $w$. This tracker does not rely on any of the assumptions relating to constant energy and velocity that followed in the derivation of the Hamiltonian, and can be used as an indicator of the accuracy of the Hamiltonian under a given set of parameters (for example initial energy and accelerating gradient). A comparison between the Hamiltonian and the tracking code for a $2 \mathrm{GeV}$ proton with $E_{0}=10 \mathrm{kV} / \mathrm{m}$ can be found in Fig. 2. The phase width of the separatrix was in excellent agreement between the Hamiltonian and the particle tracker, and the amplitude of the rf bucket at $\phi=\phi_{s}$ was within $0.5 \%$ at the peak value between the two cases.

A Hamiltonian inherently makes an assumption that $\beta_{s} \gamma_{s}=C$, where $C$ is a constant. While this is not true in the

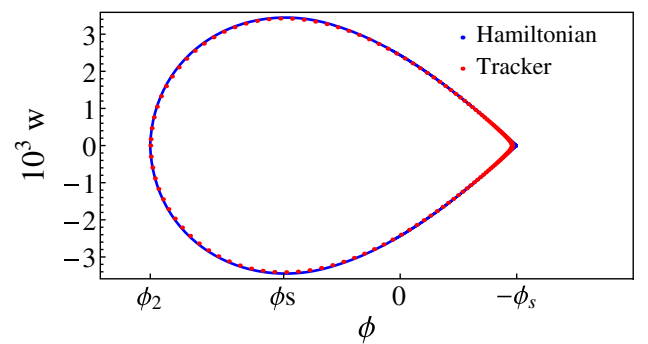

FIG. 2. Comparison of the Hamiltonian found in Eq. (14) with results from a particle tracker based on the fundamental principle of the energy gain of a particle as it traverses a cavity, found in Eq. (7). The peak amplitude is in agreement to within $0.5 \%$.

case of linear accelerators where moderate to high gradients are required, the separatrix for the case with little to no acceleration offers insight into the particle dynamics, and reveals some important physics of the longitudinal phase space. Some of the unique features found with no acceleration can then be seen in cases when high accelerating gradients are included.

We will now discuss the validity of these approximations and calculate the Jacobian determinant to verify the accuracy of the linear map [18].

The Jacobian determinant provides the phase space area preserving property (or a magnification factor for the single particle emittance) and is equal to unity for any particle transformation with a constant velocity. However when acceleration is present a deviation from unity occurs, which arises from the thin lens model that implements a momentum impulse at the position of the cavity. This is because the particle is gaining energy and therefore the velocity changes either side of the gap. It is important to calculate the magnitude of this deviation in order to ensure the linear map is accurate. This can be calculated for a transformation either side of the thin lens for a drift-kick-drift model. Here, the phase before and after remains the same, $\phi_{f}=\phi_{i}$ while the energy is increased after crossing the gap. This gives the final energy as $W_{f}=W_{i}+q g E_{1} T(\beta) \cos \left(\phi_{i}\right)$. The Jacobian determinant can be shown to be

$$
\begin{aligned}
\frac{\partial\left(W_{f}, \phi_{f}\right)}{\partial\left(W_{i}, \phi_{i}\right)} & =\left|\begin{array}{ll}
\frac{\partial W_{f}}{\partial W_{i}} & \frac{\partial \phi_{f}}{\partial W_{i}} \\
\frac{\partial W_{f}}{\partial \phi_{i}} & \frac{\partial \phi_{f}}{\partial \phi_{i}}
\end{array}\right| \\
& =1-\frac{q g E_{1}}{2 W_{i}} k T^{\prime}(k) \cos \left(\phi_{i}\right),
\end{aligned}
$$

where $T^{\prime}(k)=\frac{d T(k)}{d k}$. The determinant is clearly equal to unity for no acceleration and it can be seen that when acceleration is present the determinant deviates from unity. This is not an issue for accurately calculating the trace of a particle through the linac, as long as this deviation is acceptably small. There are two ways of reducing this deviation, either the acceleration gradient is reduced, or the 
initial energy of the particle is increased. This allows the determinant to be very close to unity, which allows the phase space behavior of particles to be accurately modeled in the regime where moderate gradients are present. As seen in Ref. [18], a phase correction can be applied that can correct for any error that occurs when crossing the boundary, however for the regimes which are being discussed here this is not needed. For example, with an initial energy in the region of 6 times the rest mass of the particle being used, the discrepancy of Eq. (16) is $\mathcal{O}\left(10^{-3}\right)$ for $10 \mathrm{kV} / \mathrm{m}$.

When moderate or high acceleration is present, $\beta \rightarrow 1$ and $\frac{d \beta}{d W} \rightarrow 0$. This causes adiabatic phase damping (due to the slower response of the particle in the phase coordinate) which results in larger energy spreads. This behavior is a consequence of the kinetic energy term in the Hamiltonian. As $\beta_{s} \gamma_{s}$ increases, the kinetic term of the Hamiltonian is decreasing. Due to the changing value of the Hamiltonian, there is no longer a contour that represents the boundary between stable and unstable orbits [18]. Instead the input acceptance is calculated, which specifies the initial positions for an ensemble of particles that will be stable. The input acceptance is a boundary in phase space for the initial coordinates of a collection of particles. If a particle's initial coordinates are within the input acceptance, then the particle will undergo a stable orbit and be captured by the bucket. This effect is shown by the spiral-like trajectories seen in Fig. 3. The particle undergoes phase damping, which results in a growth on the energy axis. It is also possible for particles that have very high initial phase and energy values to be captured by the bucket because the tail from the separatrix extends until high values. A particle with an initial position inside this tail will take a long time to reach the bucket, but will eventually be captured with the rest of the bunch. This type of behavior is unique to linacs and has been seen at many different facilities [20].

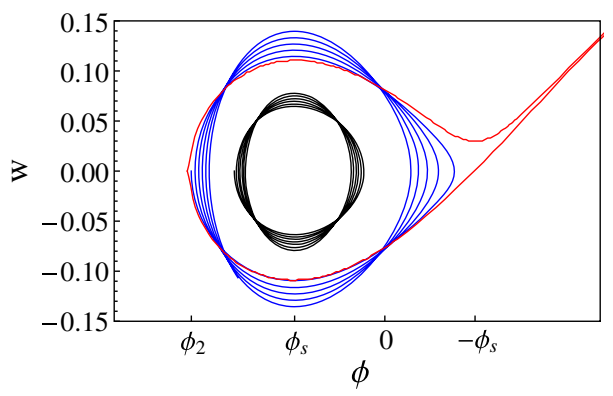

FIG. 3. A single input acceptance is shown for $E_{0}=10 \mathrm{MV} / \mathrm{m}$. The red curve is the boundary of the input acceptance and the blue and black curves are particle trajectories with different initial positions. The trajectory can move beyond the boundary as the boundary only applies to the initial position of the particles. The spiral nature is caused by the adiabatic phase damping due to the increasing $\beta_{s} \gamma_{s}$.
A Hamiltonian that describes the motion in phase space of a particle as it traverses through a cavity oscillating in a single mode has been derived. In the next section, this formalism will be extended to allow the modeling of two harmonically related modes with different longitudinal field profiles.

\section{HAMILTONIAN FOR MULTIHARMONIC CAVITIES}

Here, a Hamiltonian is derived that facilitates an arbitrary variation of the fundamental mode with an additional harmonic. The electric field in a multiharmonic cavity can be generalized as

$$
\begin{aligned}
E_{T}= & (1-\alpha) E_{z 1}(z) \cos (\omega t+\phi) \\
& +\alpha E_{z h}(h, z) \cos \left[h(\omega t+\phi)+\phi_{n h}\right],
\end{aligned}
$$

where $h$ is the harmonic number of the mode, $\phi_{n h}$ is the phase shift for the time-dependent component of the harmonic mode, $\alpha$ is the percentage mode contribution from the harmonic mode and $E_{z 1}(z)$ and $E_{z h}(h, z)$ are the longitudinal field profiles for the fundamental and harmonic modes respectively. The energy gain of a particle traversing this field is given by

$$
\begin{aligned}
W= & q\left[(1-\alpha) \int_{-g / 2}^{g / 2} E_{z 1}(z)[\cos (\omega t) \cos (\phi)\right. \\
& -\sin (\omega t) \sin (\phi)] d z \\
& +\alpha \int_{-g / 2}^{g / 2} E_{z h}(h, z)\left[\cos (h \phi) \cos \left(h \omega t+\phi_{n h}\right)\right. \\
& \left.\left.-\sin (h \phi) \sin \left(h \omega t+\phi_{n h}\right)\right] d z\right],
\end{aligned}
$$

which can be simplified to

$$
\begin{aligned}
W= & q\left\{(1-\alpha)\left[V_{1} \cos (\phi)-V_{2} \sin \phi\right]\right. \\
& \left.+\alpha\left[V_{h 1} \cos (h \phi)-V_{h 2} \sin (h \phi)\right]\right\},
\end{aligned}
$$

where

$$
\begin{aligned}
V_{1} & =\int_{-g / 2}^{g / 2} E_{z 1}(z) \cos \left(\omega \frac{z}{\beta c}\right) d z, \\
V_{2} & =\int_{-g / 2}^{g / 2} E_{z 1}(z) \sin \left(\omega \frac{z}{\beta c}\right) d z, \\
V_{h 1} & =\int_{-g / 2}^{g / 2} E_{z h}(h, z) \cos \left(h \omega \frac{z}{\beta c}+\phi_{n h}\right) d z, \\
V_{h 2} & =\int_{-g / 2}^{g / 2} E_{z h}(h, z) \sin \left(h \omega \frac{z}{\beta c}+\phi_{n h}\right) d z .
\end{aligned}
$$

The difference equation for the energy gain can now be given as 


$$
\begin{aligned}
\Delta W_{n}= & q W_{0}\left[( 1 - \alpha ) \left(V_{1}\left[\cos (\phi)-\cos \left(\phi_{s}\right)\right]\right.\right. \\
& \left.-V_{2}\left[\sin (\phi)-\sin \left(\phi_{s}\right)\right]\right) \\
& +\alpha\left[V_{h 1}\left(\cos (h \phi)-\cos \left(h \phi_{s}\right)\right)\right. \\
& \left.\left.-V_{h 2}\left(\sin (h \phi)-\sin \left(h \phi_{s}\right)\right)\right]\right],
\end{aligned}
$$

where $W_{0}$ is a scaling factor introduced to ensure the energy gain of the synchronous particle is the same for each variation within each study.

As described in the previous section, one can now move from discrete cavity gaps to continuous longitudinal coordinates, using $d n=d s / g$ and $w=\left(W-W_{s}\right) / m c^{2}$, this allows the coupled equations to be obtained as

$$
\begin{aligned}
\frac{d w}{d s}= & \frac{q}{g m c^{2}}\left[( 1 - \alpha ) \left(V_{1}\left[\cos (\phi)-\cos \left(\phi_{s}\right)\right]\right.\right. \\
& \left.-V_{2}\left[\sin (\phi)-\sin \left(\phi_{s}\right)\right]\right) \\
& +\alpha\left[V_{h 1}\left(\cos (h \phi)-\cos \left(h \phi_{s}\right)\right)\right. \\
& \left.\left.-V_{h 2}\left(\sin (h \phi)-\sin \left(h \phi_{s}\right)\right)\right]\right]=-\frac{\partial H}{\partial \phi},
\end{aligned}
$$

and

$$
\frac{d \phi}{d s}=-2 \pi \frac{w}{\gamma_{s}^{3} \beta_{s}^{3} \lambda}=\frac{\partial H}{\partial w} .
$$

The latter is unchanged from the single mode case.

By following the same procedure as found in the previous section, a Hamiltonian that can be used to explore several different regimes for a multiharmonic rf cavity can be found and is given by

$$
\begin{aligned}
H= & -\frac{\pi}{\beta_{s}^{3} \gamma_{s}^{3} \lambda} w^{2}-\frac{q W_{0}}{g m c^{2}}\left[( 1 - \alpha ) \left(V_{1}\left[\sin (\phi)-\phi \cos \left(\phi_{s}\right)\right]\right.\right. \\
& \left.+V_{2}\left[\cos (\phi)+\phi \sin \left(\phi_{s}\right)\right]\right) \\
& +\alpha\left(V_{h 1}\left[\frac{\sin (h \phi)}{h}-\phi \cos \left(h \phi_{s}\right)\right]\right. \\
& \left.\left.+V_{h 2}\left[\frac{\cos (h \phi)}{h}+\phi \sin \left(h \phi_{s}\right)\right]\right)\right] .
\end{aligned}
$$

The kinetic energy term remains unchanged from the single mode case; this is because if a particle moves away from the synchronous particle by $d \phi$ in terms of the fundamental, then the movement for the additional harmonic will be $h d \phi$, which is taken into account in the potential. The Hamiltonian simplifies when a particular longitudinal profile is specified. For $E_{z 1}(z)=$ $E_{0} \cos (k z)$, i.e. an even function, then $V_{2}=0$ and $V_{1}=\int_{-g / 2}^{g / 2} E_{0} \cos (k z) \cos \left(\frac{\omega z}{\beta c}\right) d z$, whereas a reverse effect happens for an odd longitudinal function. Similarly, this also occurs for the harmonic mode when $\phi_{n h}=0$.
Depending on whether $E_{z h}(h, z)$ is even or odd, $V_{h 1}$ or $V_{h 2}$ becomes 0 respectively. However, for cases when $\phi_{n h} \neq 0$, this simplification does not occur.

The synchronous phase in a multiharmonic cavity becomes more difficult to select as there are now two independent modes in the cavity, and the contribution from each one is dependent on its specific parameters. In order to remain consistent between the treatment of each set of parameters, a new method is used that allows an appropriate synchronous phase to be determined. First, a synchronous phase $\Phi_{s}$ is chosen which, in the single mode case, would correspond to a reduction in the peak accelerating gradient of $\cos \Phi_{s}$. Equation (19) is then solved for $\phi$ to determine the peak energy gain in the cavity, $W_{\max }$. The synchronous phase is then selected as the phase required to reduce this peak gradient by $\cos \Phi_{s}$, i.e. such that the energy gain of the synchronous particle, $W_{s}$, is given by $W_{s}=W_{\max } \cos \Phi_{s}$. Typically, we will use $\Phi_{s}=-\pi / 3$, which results in a gradient reduction of $50 \%$. This is much larger than is typically used in a practical accelerator, but it allows a greater insight into the physics of the longitudinal dynamics for each case as it increases the phase width of the rf bucket.

The linear map for this Hamiltonian is similar to the single mode case, with an extension to include the additional mode. As before, the phases before and after the transformation are the same, i.e. $\phi_{f}=\phi_{i}$, the energy transformation is now

$$
\begin{aligned}
W_{f}= & W_{i}+q\left\{(1-\alpha)\left[V_{1}(k) \cos \left(\phi_{i}\right)-V_{2}(k) \sin \left(\phi_{i}\right)\right]\right. \\
& \left.+\alpha\left[V_{h 1}(k) \cos \left(h \phi_{i}\right)-V_{h 2}(k) \sin \left(h \phi_{i}\right)\right]\right\}
\end{aligned}
$$

The Jacobian determinant of the Hamiltonian is given by

$$
\begin{aligned}
\frac{\partial\left(W_{f}, \phi_{f}\right)}{\partial\left(W_{i}, \phi_{i}\right)} & =\left|\begin{array}{ll}
\frac{\partial W_{f}}{\partial W_{i}} \frac{\partial \phi_{f}}{\partial W_{i}} \\
\frac{\partial W_{f}}{\partial \phi_{i}} \frac{\partial \phi_{f}}{\partial \phi_{i}}
\end{array}\right| \\
& =1-\frac{q k}{2 W_{i}}\left[(1-\alpha)\left(V_{1}^{\prime}(k) \cos \left(\phi_{i}\right)-V_{2}^{\prime}(k) \sin \left(\phi_{i}\right)\right)\right. \\
& \left.+\alpha\left(V_{h 1}^{\prime}(k) \cos \left(h \phi_{i}\right)-V_{h 2}^{\prime}(k) \sin \left(h \phi_{i}\right)\right)\right],
\end{aligned}
$$

where $V^{\prime}(k)=d V / d k$. The derivative of the harmonic voltages gives rise to an $h$ factor from the longitudinal profile. This results in the harmonic mode being more sensitive to errors in the linear map. Calculations have shown that perturbations arising from this determinant are of the same order as the single mode case (for the same initial energy and accelerating gradient). Therefore, assuming appropriate bunch parameters are chosen, no issue is anticipated.

We now apply Eq. (27) to glean some insight into the beam dynamics in second harmonic cavities. 


\section{SECOND HARMONIC CAVITY}

When considering a second harmonic cavity that excites $\mathrm{TM}_{010}$ and $\mathrm{TM}_{011}$ modes simultaneously, the harmonic number is $h=2$ and $E_{z h}(h, z)=E_{0} \sin (h k z)$.

Here, $\phi_{n h}$ is varied from 0 to $\frac{\pi}{2}$ and the acceptance of the rf bucket is determined for each step. Throughout the variation of $\phi_{n h}$, a constant value of $\alpha=0.222$ is used. This value arises from an optimization of a second harmonic cavity suitable for beam driven applications [12]. The amplitude of the fundamental and harmonic mode is kept constant at $10 \mathrm{kV} / \mathrm{m}$, and the total energy gain across the cavity is determined by $(1-\alpha) W_{1}+\alpha W_{2}$. The synchronous phase is selected as the phase that gives a reduction in the total gradient corresponding to $\Phi_{s}=-\pi / 3$, with the synchronous phase being recalculated for each step as described in Sec. III.

As discussed before, two cases will be shown: one for small accelerating gradients of $10 \mathrm{kV} / \mathrm{m}$ and one for high accelerating gradients of $10 \mathrm{MV} / \mathrm{m}$.

The particle trajectories are solved for different initial conditions for a proton with an initial energy $W_{i}=7 \mathrm{~m}$, where $m$ is the proton rest mass. These plots are shown for different values of $\phi_{n h}$ in Fig. 4.
For $\phi_{n h}=0$, a slight distortion in the energy gain as a function of $\phi$ is observed around $\phi=\pi / 2$. In the region of the synchronous phase however, the energy gain is approximately sinusoidal. This is why the shape of the separatrix does not deviate significantly from the single mode case. When $\phi_{n h}$ increases, the energy gain of a particle in the region of $\phi>0$ begins to increase and draw level with the energy gain at $\phi<0$. This culminates in a flattening of the bucket at $\phi_{n h}=0.5 \pi$ around $\phi=0$. The bucket is now much wider, and is flatter at the highest energy excursion. This flattening is similar to some of the results obtained at the PSB [14] and ELETTRA [15] where this type of behavior can increase the lifetime of the beam.

The acceptance of each rf bucket can be found by numerically calculating the area of the separatrix. This is plotted in Fig. 5. It can be seen that initially, the acceptance is reducing because the gradient of the energy gain vs phase is reducing for the region around the synchronous particle. However as the bucket begins to flatten, the phase width increases which causes the acceptance of the bucket to rise up.

In order to verify the plots found in Fig. 4, single particle tracking was performed and compared with the Hamiltonian for $\phi_{n h}=\frac{\pi}{2}$. The reason for choosing this
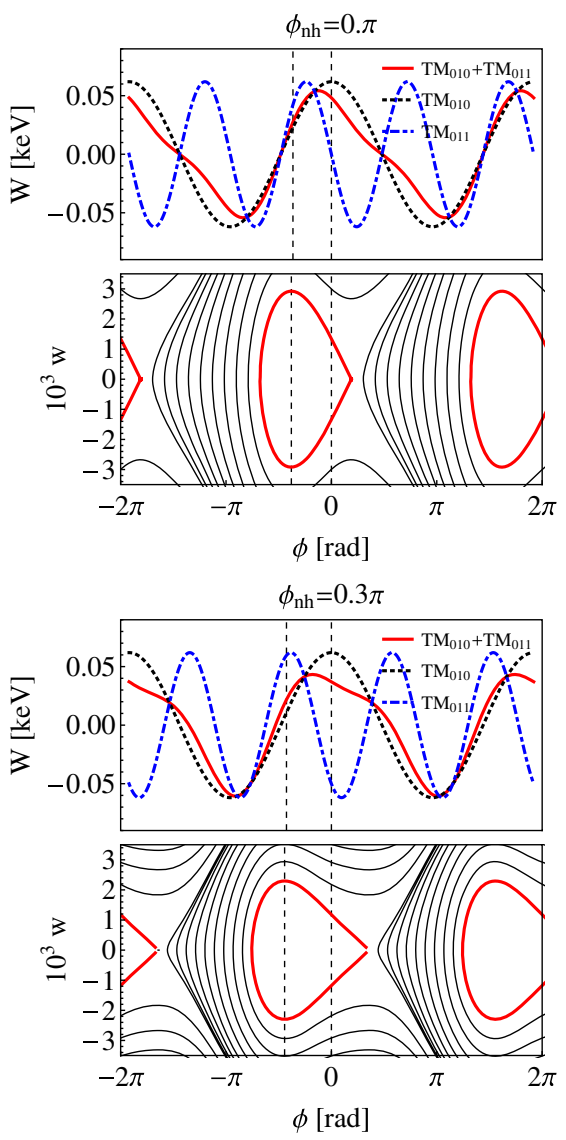
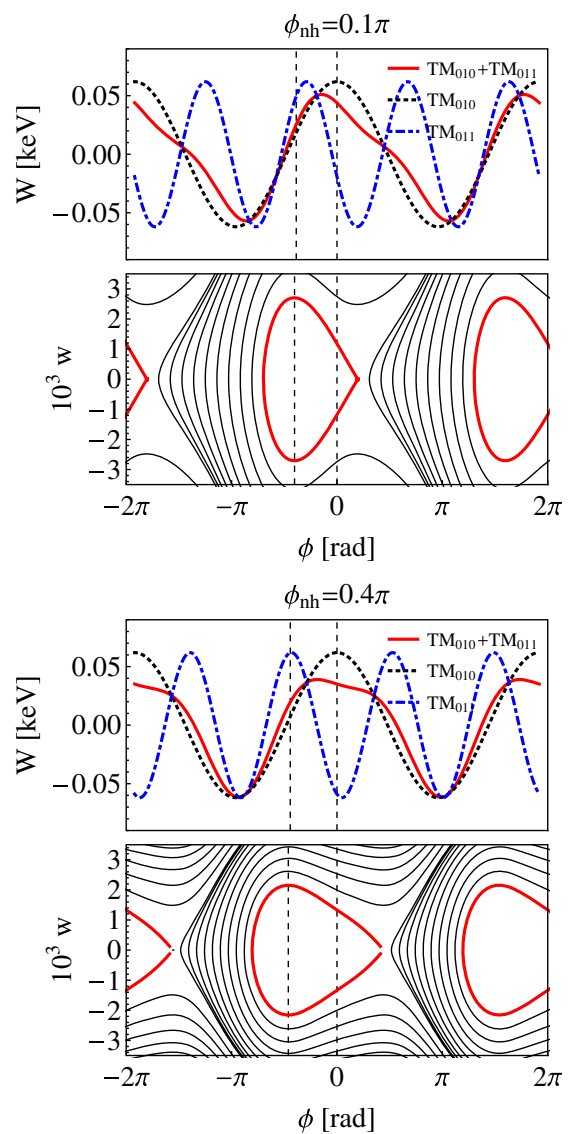
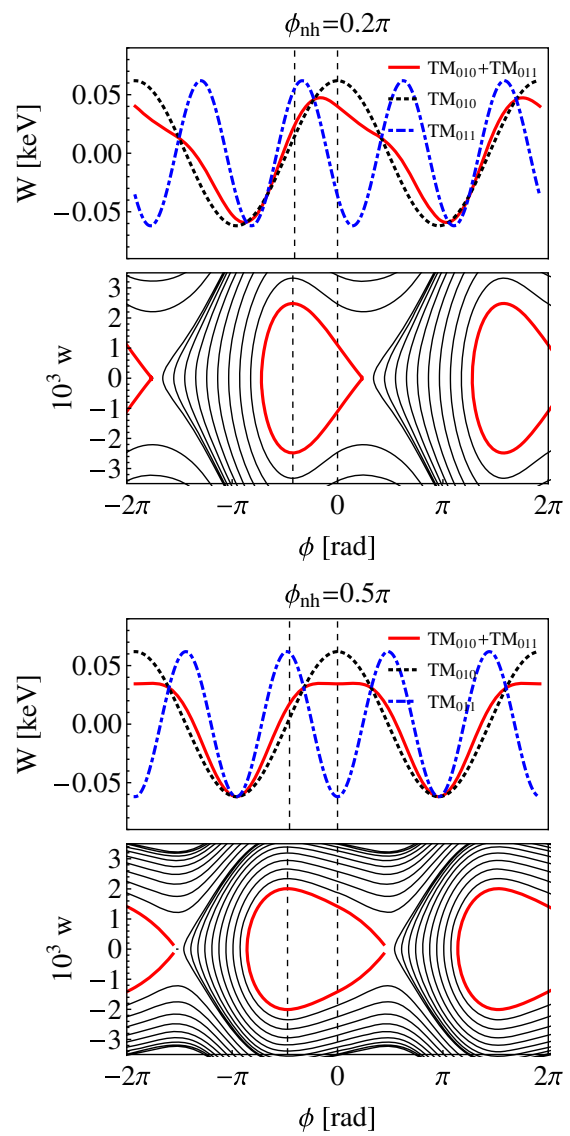

FIG. 4. Phase space plots for a cavity exciting both the $\mathrm{TM}_{010}$ and $\mathrm{TM}_{011}$ modes. A phase shift is gradually applied to the $\mathrm{TM}$.11 mode and the gradient is kept constant for each step. For all steps, $E_{0}=10 \mathrm{kV} / \mathrm{m}$. The upper plot in each step is the energy gain over the cavity as a function of $\phi$ and the bottom is the phase space plots, where the red line marks the separatrix. 


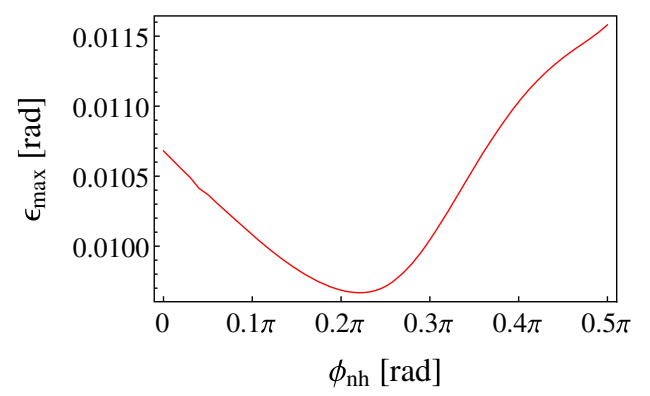

FIG. 5. The normalized acceptance of the rf bucket vs the phase shift of the $\mathrm{TM}_{011}$ mode.

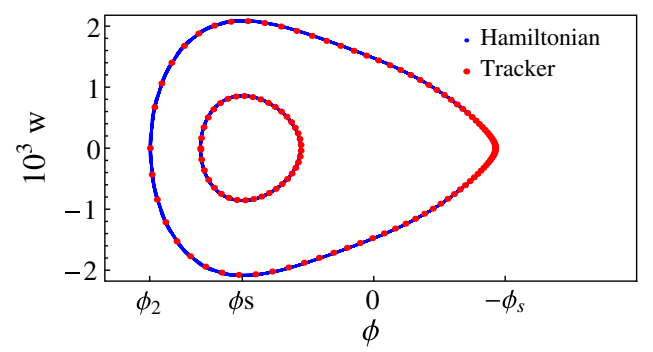

FIG. 6. Comparison between particle tracking based on fundamental principles with the Hamiltonian from Eq. (27) for $\phi_{n h}=0.5 \pi$. The larger contour is for the separatrix, with the smaller trace showing a small phase deviation.

particular step is because it is the most distinctive plot and differs the most from a single mode separatrix. The results from the tracking can be found in Fig. 6. The tracking was found to be in excellent agreement, with the phase behavior perfectly matching the Hamiltonian and an amplitude difference of less than $0.5 \%$, very similar to what was seen in the single mode case.
We now model the same set of cavity parameters for the case when $E_{0}=10 \mathrm{MV} / \mathrm{m}$. The input acceptance is calculated for each step and the main features from each of the graphs shown in Fig. 7 are still present. Here, the spiral-like trajectories are omitted from the figures as they do not provide any new insight into the dynamics. It can be seen that as the energy gain is flattening in the region around $\phi=0$ for $\phi_{n h} \rightarrow 0.5 \pi$, the input acceptance begins to distort and expand around $-\phi_{s}$. In the plots, the point $\phi_{2}$ refers to the position of the edge of the separatrix from the case without acceleration. This is to show any additional phase width that may be gained by including acceleration. The area of the input acceptance behaves the same in the case with acceleration as it does without acceleration, while also retaining the same key features observed.

Having modeled the acceptance of the rf bucket in a cavity that can excite a fundamental and second harmonic mode, we now apply the procedure to an ESS-like linac.

\section{APPLICATION OF HAMILTONIAN TO A MODIFIED ESS LINAC}

We now consider the application of these techniques to a specific accelerator. We will show that a multiharmonic cavity is capable of accelerating bunches that are provided by the injection facility at the European Spallation Source (ESS). The ESS will be a facility that will produce neutron beams of unparalleled brightness via spallation [21]. This will be achieved by accelerating protons up to $2.5 \mathrm{GeV}$ and colliding with a rotating tungsten target. The full proton linac is comprised of several different types of cavities (as $\beta$ is increasing throughout the linac) which operate with different frequencies. Here we investigate the implications on longitudinal phase space of replacing the single mode
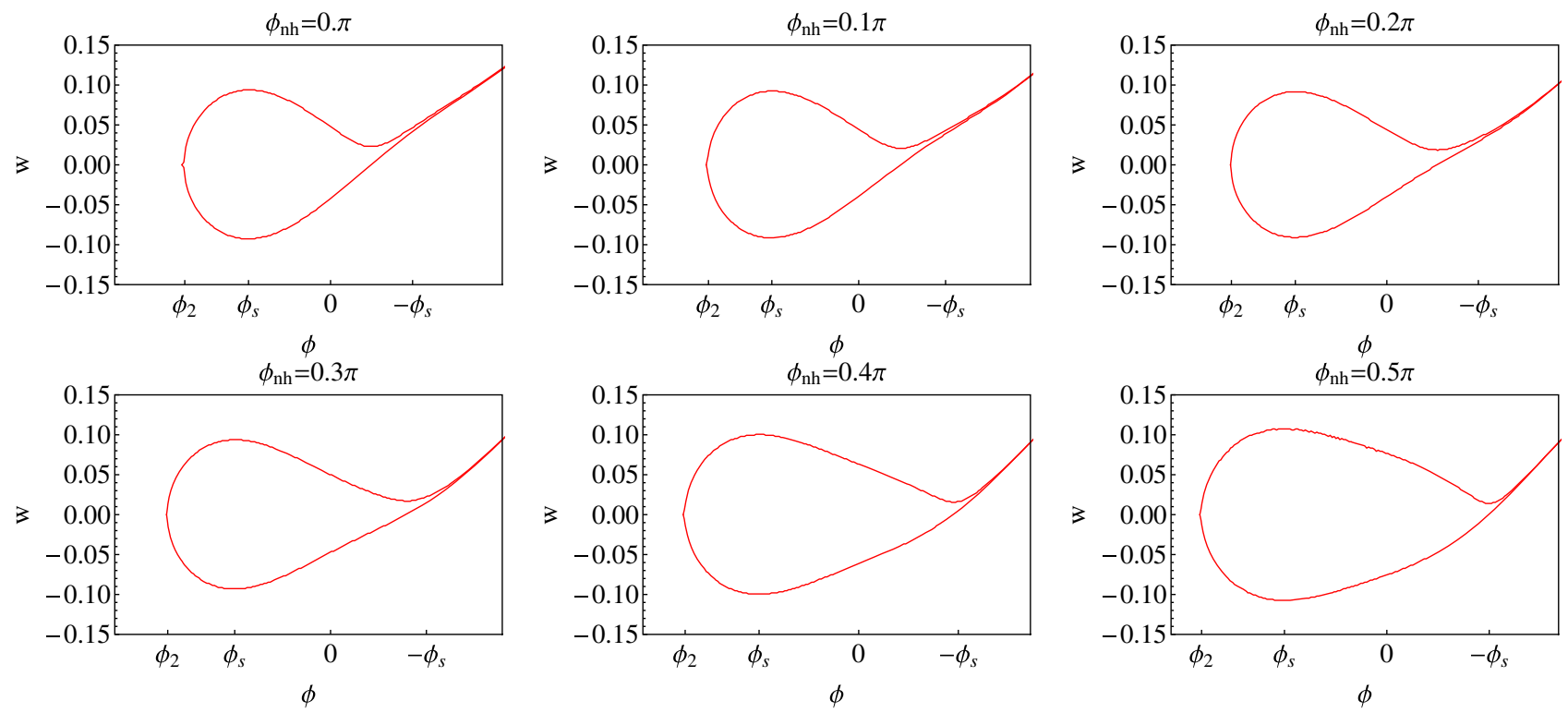

FIG. 7. Input acceptance for a $\mathrm{TM}_{011}$ second harmonic cavity for $E_{0}=10 \mathrm{MV} / \mathrm{m}$ and an initial energy $W_{i}=7 m$, where $m$ is the proton rest mass. In each case, $\phi_{2}$ corresponds to respective $\phi_{2}$ found from the case where no acceleration is present. 
cavities with multiharmonic cavities. In order to make this analysis feasible, we will make some broad simplifications on the composition of the linacs. This approach will be made clear once the characteristics of the linac are described.

The proton accelerator consists of eight different sections, which include transfer lines and radio-frequency quadrupoles (RFQ) at low energy, and then several different types of cavities for higher energy acceleration. The drift tube linac (DTL) and Spoke cavities operate at $352.2 \mathrm{MHz}$, with an accelerating voltage of approximately $6 \mathrm{MV}$ (see Fig. 4.15 in [21]). The protons are then accelerated by a set of $704.4 \mathrm{MHz}$ superconducting cavities, which operate with an accelerating gradient of $18 \mathrm{MV} / \mathrm{m}$. As most of the energy gain occurs in these high- $\beta$ cavities, we will approximate the ESS linac as one continuous structure of identical cavities, all operating with frequency 704.4 MHz and $18 \mathrm{MV} / \mathrm{m}$ accelerating gradient. This major simplification allows a direct comparison to be made between the single mode and multiharmonic features and we refer to this situation as an equivalent linac.

In each case, the input acceptance will be computed for the equivalent linac. This is from the tracking of many individual particles along the linac. These will then be overlaid with a distribution corresponding to the anticipated bunch parameters that are used as the input for the DTL section of the ESS (Fig. 4.4 in [21]). It can be seen that, at the end of the RFQ (but before the injection into the high- $\beta$ cavities), the $5 \sigma$ energy spread is approximately $0.05 \mathrm{MeV}$, and the $5 \sigma$ phase spread is approximately 0.2 radians (at $704.4 \mathrm{MHz}$ ). For simplicity, the bunch is represented as a Gaussian distribution which is truncated at $5 \sigma$ in both dimensions.

First, we consider the beam dynamics in the equivalent linac for the single mode case. For the cavities in the high- $\beta$ linac, the synchronous phase $\phi_{s}=-\pi / 12$, we therefore use the same synchronous phase for this case. Figure 4.14 in Ref. [21] shows the input acceptance for the ESS linac, starting from the DTL. We will first calculate the input acceptance for the equivalent linac.

We then turn our attention to adding multiharmonic cavities in place of the main accelerating cavities in the equivalent linac. Multiharmonic cavities allow higher gradients to be achieved without increasing the effect of the intense surface electromagnetic fields. In order to investigate this effect we maintain an accelerating gradient of $18 \mathrm{MV} / \mathrm{m}$ from the fundamental mode, and add an additional second harmonic field component and evaluate the acceptance of the equivalent linac. We choose similar parameters to that used in Sec. IV, $h=2, \alpha=0.222$ and $\phi_{n h}=\pi / 2$, and adjust the synchronous phase such that the equivalent reduction in energy gain corresponds to $\Phi_{s}=-\pi / 12$ (following the same procedure as used elsewhere in this study). This is also shown with the same input bunch distribution, but now it has been shifted to account for the change in synchronous phase. The results from these simulations are displayed in Fig. 8.

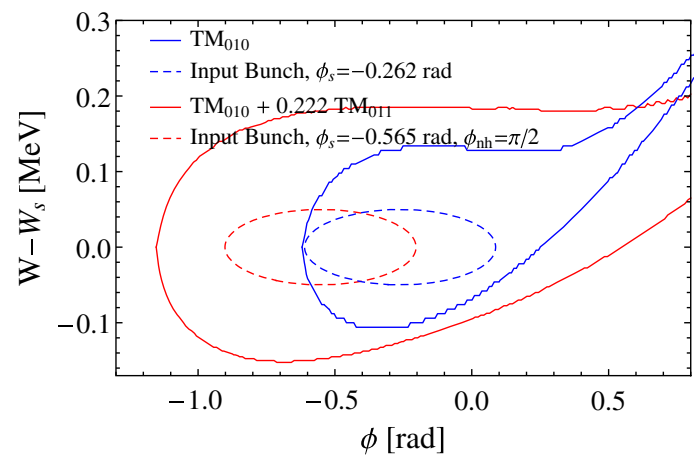

FIG. 8. Input acceptance for an equivalent ESS linac for a fundamental mode alone (blue) and for a fundamental mode in addition to a second harmonic mode (red). The dashed line represents the input bunch into the equivalent from the end of the RFQ section of the ESS. The initial phases of the input bunches have been shifted to reflect the synchronous phase of each setup.

The input acceptance for the single mode equivalent linac is in reasonable agreement with the full tracking used for the ESS linac [21]. It can also be seen that the input bunch readily fits inside the input acceptance, showing that the input distribution truncated at $5 \sigma$ could be accelerated in a stable manner with minimal losses.

When introducing a second harmonic in addition to the fundamental mode, it can be seen that (for the parameters specified) the bucket width and bucket height both increase. The input bunch distribution is fully enveloped by the input acceptance, with more margin for error than what would be expected for the single mode case.

In order to compare the output bunch parameters of the equivalent linac, 5000 particles were tracked throughout the linac for both single mode and multimode cases. The relevant histograms for both the phase and energy distributions can be found in Figs. 9 and 10.

It can be seen that the output phase distribution is very similar for both cases ( 0.86 mrads for single mode vs 0.88 mrads for multimode). This can be expected because

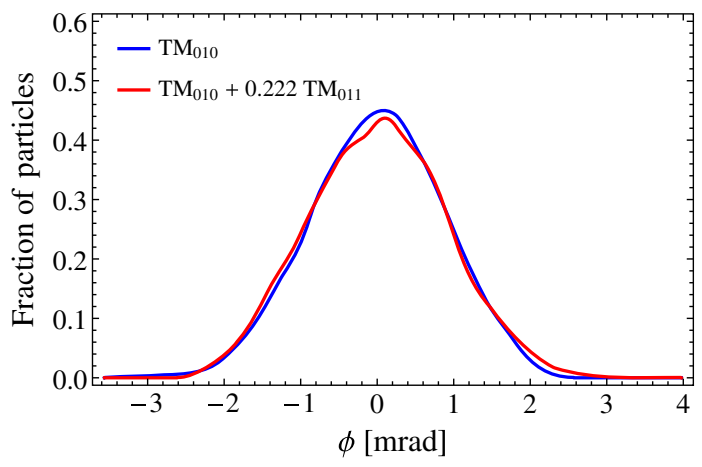

FIG. 9. Phase distribution for the output bunch from the equivalent linac for single mode and multimode cases. The phases have been shifted by their synchronous phases such that they are centered on 0 . 


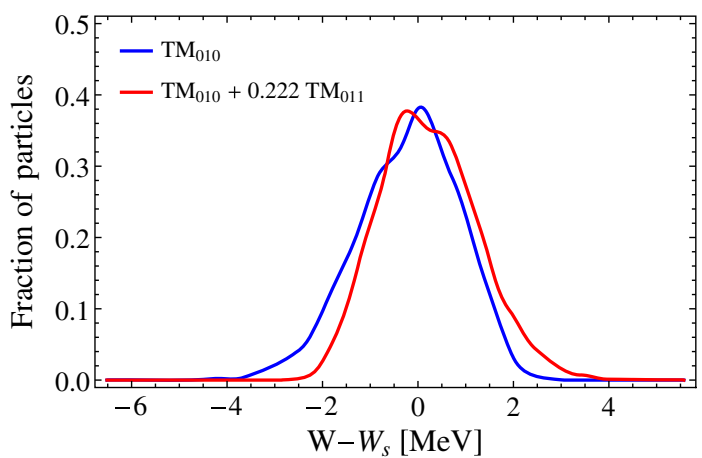

FIG. 10. Energy distribution for the output bunch from the equivalent linac for single mode and multimode cases.

both distributions comfortably fit inside the input acceptance. Note that for this plot both curves have been shifted by their respective synchronous phases. For the energy distribution, it can again be seen that the original single mode case is very similar to the multimode case (standard deviation of 1.07 for single mode vs 1.00 for multimode), except now there is a small asymmetry between negative and positive energy deviations.

The aim of these calculations was to show that a multiharmonic cavity could be used to accelerate bunches with realistic phase space parameters. It is clear that, for optimized parameters of the additional harmonic, multimode cavities could be used for the acceleration and transport of bunches from medium to high energies.

\section{CONCLUSION}

We have derived a Hamiltonian in order to study the longitudinal stability of charged particle beams. In addition to the usual single mode beam dynamics, our methodology is sufficiently general to accommodate additional modes. The derivation of this Hamiltonian is based upon the standard formalism found in the literature [18], which we extend to allow for different longitudinal profiles. We added a harmonic of the fundamental accelerating mode, and investigated if the beam dynamics was appreciably affected, and indeed if the beam remained phase stable. This Hamiltonian can be applied to a variety of different types of multiharmonic cavity, ranging from rf driven to beam driven cavities, and could have potential applications in proton or heavy ion linacs, or to multiharmonic cavities that operate in the two beam acceleration scheme [4].

We have explored the fundamental characteristics of acceptance for cavities in which a second harmonic of the main accelerating mode has been independently added. Furthermore, we considered the beam dynamics that would occur in a proton linac with broadly similar characteristics to that of the superconducting cavities in the main ESS linac; albeit with some significant simplifying assumptions in order to assess the main characteristics of the particles in phase space and with no attempt to redesign the cavities with a view to increasing the overall accelerating field. The beam dynamics behavior, as predicted from simulations, showed that the addition of a second harmonic can enhance the overall acceptance. Thus, from the perspective of longitudinal phase space alone, an addition of a harmonic mode to the main accelerating mode is quite feasible.

This is the first analysis, to the knowledge of the authors, which considers this aspect of longitudinal stability of charged particles in multiharmonic cavities. The fabrication and testing of these types of cavities is ongoing at the Yale Beam Physics Laboratory [12]. However, the impact of phase space beam dynamics is likely to be assessed at a somewhat later stage as at present the focus is on the implications on raising the accelerating field, whilst lowering the breakdown threshold and the effect from pulsed surface heating [10]. For this reason comparison of experiments to these phase space simulations is not likely to occur in the near future.

\section{ACKNOWLEDGMENTS}

The authors would like to thank A. Wolski for discussions on aspects of Hamiltonian dynamics. L. C. received funding as part of an STFC Cockcroft Institute studentship. This material is based upon work supported by the U.S. Department of Energy, Office of Science, Office of High Energy Physics, under Award No. DE-SC0010699.

[1] S. T. Heikkinen, S. Calatroni, and H. Neupert, CERN Report No. CERN-OPEN-2006-004, 2006.

[2] W. Wuensch, C. Achard, S. Döbert, H. Braun, I. Syrachev, M. Taborelli, and I. Wilson, Demonstration of highgradient acceleration, in Proceedings of the Particle Accelerator Conference, Portland (IEEE, New York, 2003), Vol. 1, pp. 495-497.

[3] W. Wuensch, Progress in understanding the high-gradient limitations of acceleration structures, in Proceedings of the 4th Asian Particle Accelerator Conference, Indore, 2007 (RRCAT, Indore, 2007).

[4] S. Y. Kazakov, S. V. Kuzikov, Y. Jiang, and J. L. Hirshfield, High-gradient two-beam accelerator structure, Phys. Rev. ST Accel. Beams 13, 071303 (2010).

[5] H. Braun et al., CERN Report No. CERN-OPEN-2008021, 2008.

[6] J.P. Delahaye et al., CLIC: A two beam multi-TeV e+- linear collider, eConf C000821, MO201 (2000).

[7] A. Grudiev, D. Schulte, and W. Wuensch, Optimum frequency and gradient for the CLIC main linac accelerating structure, Conf. Proc. C060626, 1867 (2006).

[8] R. M. Jones, Wakefield suppression in high gradient linacs for lepton linear colliders, Phys. Rev. ST Accel. Beams 12, 104801 (2009).

[9] D. Pritzkau, Ph.D. thesis, Stanford University, 2001.

[10] S. V. Kuzikov, S. Y. Kazakov, Y. Jiang, and J. L. Hirshfield, Asymmetric Bimodal Accelerator Cavity for Raising RF 
Breakdown Thresholds, Phys. Rev. Lett. 104, 214801 (2010).

[11] Y. Jiang, S. V. Kuzikov, S. Y. Kazakov, and J. L. Hirshfield, Multiharmonic test setup for RF breakdown studies, Nucl. Instrum. Methods Phys. Res., Sect. A 657, 71 (2011).

[12] Y. Jiang and J. L. Hirshfield, Multiharmonic accelerating cavities for $\mathrm{rf}$ breakdown studies, in Proceedings of the Particle Accelerator Conference, Pasadena (2013), WEPMA28, p. 1041.

[13] P. Marchand, Possible upgrading of the SLS RF system for improving the beam lifetime, in Proceedings of the 18th Particle Accelerator Conference, New York, 1999 (IEEE, New York, 1999), Vol. 2, p. 989.

[14] K. Hanke, Past and present operation of the CERN PS booster, Int. J. Mod. Phys. A 28, 1330019 (2013).

[15] M. Pedrozzi, W. Gloor et al., First operational results of the 3rd harmonic superconducting cavities in SLS and ELET-
TRA, in Proceedings of the 2003 Particle Accelerator Conference, Portland, (Ref. [2]), Vol. 2, p. 878.

[16] J. Grieser, D. Lens, U. Hartel, H. Klingbeil, U. Laier et al., A digital beam-phase control system for a heavy-ion synchrotron with a double-harmonic cavity system, in Proceedings of the International Particle Accelerator Conference, Shanghai, China (2013), WEPME004, p. 2926.

[17] E. Vogel, M. Bonezzi, A. Bosotti, M. Fusetti, P. Michelato et al., Status of the XFEL $3.9 \mathrm{GHz}$ injector section, in Proceedings of the 2nd International Particle Accelerator Conference, San Sebastián, Spain (EPS-AG, Spain, 2011), Vol. C110904, p. 289.

[18] T. Wangler, RF Linear Accelerators (Wiley-VCH, New York, 2008).

[19] A. Chao and M. Tigner, Handbook of Accelerator Physics and Engineering (World Scientific, Singapore, 1999).

[20] F. Gerigk et al., Report No. CERN-2014-007, 2014.

[21] S. Peggs et al., Report No. ESS-doc-274, 2013. 